\title{
Prolonged Intravenous Instead of Oral Antibiotics for Acute Hematogenous Osteomyelitis in Children
}

\author{
James B. Wood, MD, David P. Johnson, $\mathrm{MD}^{2}$
}

${ }^{1}$ Division of Pediatric Infectious Diseases, Department of Pediatrics, Vanderbilt University Medical Center, Nashville, Tennessee; ${ }^{2}$ Division of Hospital Medicine, Department of Pediatrics, Vanderbilt University Medical Center, Nashville, Tennessee.

The "Things We Do for No Reason" (TWDFNR) series reviews practices which have become common parts of hospital care but which may provide little value to our patients. Practices reviewed in the TWDFNR series do not represent "black and white" conclusions or clinical practice standards, but are meant as a starting place for research and active discussions among hospitalists and patients. We invite you to be part of that discussion.

A previously healthy 6-year-old boy presented to the emergency room with 3 days of right lower leg pain and fevers up to $102^{\circ} \mathrm{F}$. The leg pain had progressed until he refused to walk. The patient and family did not recall any trauma to the leg. In the emergency department, he had a blood culture drawn. Because he had elevated inflammatory markers and a negative $\mathrm{x}$-ray of his right leg, a magnetic resonance imaging scan of the right leg was obtained that revealed right tibial osteomyelitis. $\mathrm{He}$ was taken to the operating room for debridement. After obtaining blood and bone cultures, he was started on intravenous (IV) vancomycin. His blood and surgical cultures grew methicillin-resistant Staphylococcus aureus, sensitive to clindamycin. Subsequent blood cultures were negative, and his inflammatory markers trended down shortly after starting therapy. As he clinically improved, a peripherally inserted central catheter (PICC) was placed, and he was discharged home to complete a 6-week course of IV vancomycin.

\section{BACKGROUND}

Osteoarticular infections (osteomyelitis and septic arthritis) are common problems in the pediatric population, affecting $\sim 1 / 2000$ children annually and accounting for approximately $1 \%$ of all pediatric hospitalizations. ${ }^{1,2}$ Osteomyelitis can occur in children of all ages and usually requires hospitalization for

*Address for correspondence and reprint requests: James B. Wood, MD, Pediatric Infectious Diseases, Vanderbilt University Medical Center, D-7221 MCN, 1161 21st Ave. South, Nashville, TN 37232; Telephone: 615-343-2401; Fax: 615-343-9723; E-mail: james.b.wood@vanderbilt.edu

Additional Supporting Information may be found in the online version of this article.

Received: June 14, 2015; Revised: December 8, 2015; Accepted: December 18, 2015

2016 Society of Hospital Medicine DOI 10.1002/jhm.2549

Published online in Wiley Online Library (Wileyonlinelibrary.com). diagnosis and initial management. The most common mechanism of infection in children is hematogenous inoculation of the bone during an episode of bacteremia (acute hematogenous osteomyelitis), particularly in young children, due to the highly vascular nature of the developing bone. Long bones, such as the femur, tibia, and humerus, are most commonly involved. Treatment of acute osteomyelitis requires prolonged administration of antimicrobial agents. Inadequately treated osteomyelitis can result in progression to chronic infection and loss of function of the affected bone. ${ }^{3}$

\section{WHY YOU MIGHT THINK PARENTERAL ANTIBIOTICS AT DISCHARGE IS SUPERIOR TO ENTERAL THERAPY}

In the United States, a large proportion of children with hematogenous osteomyelitis are discharged from the hospital with long-term parenteral intravenous antibiotics through a PICC line. ${ }^{3}$ The medical community historically favored parenteral therapy for young children with serious bacterial infections given concerns regarding impaired enteral absorption. As a result, children with osteomyelitis were initially stabilized in the hospital and discharged with parenteral therapy through a PICC line to continue or complete care, even when the organism was susceptible to a viable oral alternative such as clindamycin or cephalexin. Recommendations regarding the safety and timing to transition to oral antibiotics have been lacking. There is also extreme variation in practice in route of administration (oral vs prolonged IV therapy) in patients being discharged from the hospital with osteomyelitis. ${ }^{3,4}$ The most recent Infectious Diseases Society of America (IDSA) guidelines do not clearly state when transition to oral antibiotics may be safe. Specifically, they state that if patients are "stable and without ongoing bacteremia," they can transition to oral therapy to complete a 4 - to 6 -week course. ${ }^{5}$

\section{WHY LONG-TERM PARENTERAL ANTIBIOTICS MAY NOT BE SUPERIOR}

The use of PICC lines has increased substantially in recent years. This has led to an increasing awareness of complications associated with PICC lines. As a result, guidelines for the appropriate use of PICC lines have been established in adults by collaborators at the University of Michigan. ${ }^{6}$ Mounting evidence 
has called into question whether longer parenteral therapy is truly a "more conservative" or "safer" approach for the treatment of osteomyelitis., $3,4-9$ Providing antibiotics via a PICC line in both the inpatient and outpatient settings may not be as benign as once accepted and may not improve outcomes in osteomyelitis as expected.

\section{Costs and Potential Harms Associated With PICC Lines}

PICC lines are known to have complications in the hospital including infection and thrombotic events, ${ }^{10}$ but these events are not isolated to the hospital setting. Multiple studies have shown outpatient PICC line complication rates ranging from $29 \%$ to $41 \%$ depending on the type of catheter, the population, and the indication for use. ${ }^{8,10-13}$ In a recently published study by Keren et al. looking specifically at children with osteomyelitis, emergency department visits and readmissions for PICC line complications occurred in $15 \%$ of patients discharged with a PICC line. ${ }^{4}$ Given the potential complications and complexity that are inherent in outpatient parenteral therapy, the ISDA has even published guidelines regarding its use. ${ }^{9}$ In addition, the cost of IV antibiotics, including administration costs, need for sedation in some children for line placement, and cost of the antibiotic itself, is significantly higher compared to oral therapy. In studies looking at early conversion to oral antibiotics versus prolonged intravenous antibiotics for complicated skin and soft tissue infections, as well as perforated appendicitis, oral antibiotics were more cost effective with an average savings of $30 \%$ to $50 \%$ and $>\$ 4000$ respectively. ${ }^{14,15}$

\section{Patient Outcomes Are Similar When Comparing Parenteral and Enteral Therapy}

In addition to increased costs and medication-related complications, treatment of osteomyelitis with parenteral antibiotics through a PICC line does not improve clinical outcomes. As early as 1997, evidence emerged that an early transition to enteral therapy for osteomyelitis in children may be safe. ${ }^{16}$ In 2010, the same group published a larger randomized study with the intent of determining overall treatment duration for osteomyelitis. This study involved 131 culture-positive cases of osteomyelitis randomized to either a "shortterm" (20 days) or "long-term" (30 days) oral antibiotics following 2 to 4 days of parenteral therapy. In this study, outcomes were favorable and similar despite such a short course of parenteral antibiotics and regardless of the overall treatment duration. ${ }^{17}$ Although the aim of this study was not to compare oral and parenteral antibiotics, all patients in this large cohort were treated successfully with early transition to oral therapy.

In 2009, Zaoutis et al. published a large, multicenter, retrospective study of 1969 children with culture- positive osteomyelitis treated with either prolonged IV therapy (defined as a central line placed before discharge) or oral therapy (no central line placed). They found a $4 \%$ incidence of treatment failure in the oral therapy group compared to a $5 \%$ incidence in the prolonged IV therapy group. They concluded that early transition to oral therapy was not associated with an increased risk of treatment failure. ${ }^{3}$

More recently, Keren et al. published a comparative effectiveness study using propensity score-based matching to adjust for confounding variables. This retrospective study included 2060 children without comorbid conditions, ages 2 months to 18 years, with both culture-positive and culture-negative acute hematogenous osteomyelitis. Propensity-based matching used logistic regression to compare patient-level characteristics including age, race, insurance, length of stay, location of infection, surgical procedures, and isolation of causative pathogens. The rates of treatment failure were nearly identical in the oral therapy $(5.0 \%)$ and PICC line $(6.0 \%)$ groups. Similarly, in across-hospital (risk difference, 0.3\% [95\% confidence interval $\{\mathrm{CI}\}:-0.1 \%$ to $2.5 \%]$ ) and within-hospital (risk difference, $0.6 \%$ [ $95 \%$ CI: $-0.2 \%$ to $3.0 \%]$ ) matched analyses, children in the oral therapy group did not have more treatment failures than those in the PICC line group. In the same comparisons, both adverse drug reactions and all treatment-related events were significantly more likely to occur in children treated with long-term parenteral antibiotics. ${ }^{4}$

Other studies have looked at the treatment of culture-negative osteoarticular infections in children and have similarly found favorable outcomes in transitioning to oral therapy after a short course of parenteral treatment. ${ }^{18}$

In short, enteral therapy has similar treatment outcomes for culture-positive and culture-negative osteomyelitis without the complications associated with parenteral treatment via a PICC line.

\section{WHEN TO CONSIDER PROLONGED PARENTERAL ANTIBIOTICS}

The studies indicating the safe transition to oral antibiotics discussed above all excluded children with certain comorbid conditions. Although this varied from study to study, exclusions were as general in some as "not previously healthy," and others were as specific as hematologic malignancies, immunocompromised states, sickle cell disease, malabsorption, and penetrating injuries. Also, although we know blood cultures obtained in children with osteomyelitis are positive in only approximately half of the patients, ${ }^{19}$ the studies cited do not contain information for their study populations regarding the duration of bacteremia or endovascular complications, such as septic thrombophlebitis, which are well described in the literature. $^{20,21}$ There are limited data on optimal treatment of children with prolonged bacteremia and endovascular 
complications. Because studies generally involved previously healthy children and do not specifically address these potential complications, the safety of early oral transition in complicated cases is not clear. The current IDSA and Red Book Committee on Infectious Diseases recommend intravenous therapy for bacteremia and endovascular infections with methicillin-resistant $S$ aureus. ${ }^{5,22}$ Clinical judgement should be used when treating children with comorbid illnesses who experience persistent bacteremia $>48$ hours, or who have endovascular complications.

\section{WHAT YOU SHOULD DO INSTEAD}

For children with acute hematogenous osteomyelitis who are either culture negative and improve on empiric therapy, or who have culture results (blood or tissue) that are susceptible to a reasonable oral antibiotic agent and who have clinical improvement on initial IV antibiotic therapy, a growing body of evidence indicates that the benefit of early transition to oral antibiotics outweighs the risks of continuing with parenteral therapy. Discharging children on oral antibiotics does not increase their risk of treatment failure but seems to decrease the risk of therapy-associated complications, including increased healthcare utilization with return visits to the emergency department or the hospital. The possible exceptions to early transition to enteral antibiotics are prolonged bacteremia or endovascular infection, though there are insufficient data in the literature indicating benefits or risks of one administration route over the other.

\section{RECOMMENDATIONS}

1. Previously healthy children with acute hematogenous osteomyelitis, without endovascular complications, should be transitioned to enteral antibiotics when they are showing signs of clinical improvement, as defined by: resolution of fever, improving physical exam, ability to take oral medications, and decreasing C-reactive protein.

2. The choice of oral antibiotics should be based on the organism's antibiotic susceptibility. If cultures are negative and the child has improved on empiric IV therapy, transition to an oral regimen with similar spectrum is acceptable.

3. Patients with acute osteomyelitis should have close follow-up after discharge from the hospital, within 1 to 2 weeks, to ensure continued improvement on therapy.

\section{CONCLUSION}

Early transition to oral antibiotics should be used in children with acute, uncomplicated osteomyelitis. A growing body of evidence shows that early transition to oral antibiotics does not increase the risk of treatment failure and can obviate the need for an outpatient PICC line. Oral antibiotics do not carry the risk of potential complications and complexity that are inherent in outpatient parenteral therapy. The transition to oral therapy should occur prior to discharge from the hospital after clinical improvement. Close follow-up is essential to ensure successful treatment in children with acute osteomyelitis.

Disclosure: Nothing to report.

Do you think this is a low-value practice? Is this truly a "Thing We Do for No Reason?" Share what you do in your practice and join in the conversation online by retweeting it on Twitter (\#TWDFNR) and liking it on Facebook. We invite you to propose ideas for other "Things We Do for No Reason” topics by emailing TWDFNR@hospitalmedicine. org.

\section{References}

1. Krogstad P. Osteomyelitis. In: Feigin RD, Cherry JD, Kaplan, SL, Demmler-Harrison, GJ, eds. Feigin and Cherry's Textbook of Pediatric Infectious Diseases. Philadelphia, PA: Saunders Elsevier; 2009.

2. Vazquez M. Osteomyelitis in children. Curr Opin Pediatr. 2002;14: 112-115.

3. Zaoutis T, Localio AR, Leckerman K, Saddlemire S, Bertoch D, Keren R. Prolonged intravenous therapy versus early transition to oral antimicrobial therapy for acute osteomyelitis in children. Pediatrics. 2009; 123:636-642.

4. Keren R, Shah SS, Srivastava R, et al.; Pediatric Research in Inpatient Settings Network. Comparative effectiveness of intravenous vs oral antibiotics for postdischarge treatment of acute osteomyelitis in children. JAMA Pediatr. 2015;169(2):120-128.

5. Liu C, Bayer A, Cosgrove SE, et al. Clinical practice guidelines by the Infectious Diseases Society of America for the treatment of methicillin-resistant staphylococcus aureus infections in adults and children: executive summary. Clin Infect Dis. 2011;52(3):285292.

6. Chopra V, Flanders SA, Saint S, et al.; Michigan Appropriateness Guide for Intravenous Catheters (MAGIC) Panel. The Michigan appropriateness guide for intravenous catheters (MAGIC): results from a multispecialty panel using the RAND/UCLA appropriateness method. Ann Intern Med. 2015;163(6 suppl):S1-S40.

7. Schroeder AR, Ralston SL. Intravenous antibiotic durations for common bacterial infections in children: when is enough enough? J Hosp Med. 2014;9(9):604-609.

8. Ruebner R, Keren R, Coffin S, Chu J, Horn D, Zaoutis TE. Complications of central venous catheters used for the treatment of acute hematogenous osteomyelitis. Pediatrics. 2006;117:1210-1215.

9. Tice AD, Rehm SJ, Dalovisio JR, et al; IDSA. Practice guidelines for outpatient parenteral antimicrobial therapy. Clin Infect Dis. 2004; 38(12):1651-1672.

10. Barrier A, Williams DJ, Connelly M, Creech CB. Frequency of Peripherally Inserted Central Catheter Complications in Children. Pediatr Infect Dis J. 2012;31(5):519-521.

11. J Jumani K, Advani S, Reich NG, Gosey L, Milstone AM. Risk factors for peripherally inserted central venous catheter complications in children. JAMA Pediatr. 2013;167(5):429-435.

12. Hussain S, Gomez MM, Wludyka P, Chiu T, Rathore $\mathrm{MH}$. Survival times and complications of catheters used for outpatient parenteral antibiotic therapy in children. Clin Pediatr (Phila). 2007;46: 247-251.

13. Van Winkle P, Whiffen T, Liu IL. Experience using peripherally inserted central venous catheters for outpatient parenteral antibiotic therapy in children at a community hospital. Pediatr Infect Dis J. 2008;27:1069-1072.

14. Stephens JM, Gao X, Patel DA, Verheggen BG, Shelbaya A, Haider S. Economic burden of inpatient and outpatient antibiotic treatment for methicillin-resistant Staphylococcus aureus complicated skin and softtissue infections: a comparison of linezolid, vancomycin, and daptomycin. Clinicoecon Outcomes Res. 2013;5:447-457.

15. Adibe OO, Barnaby K, Dobies J, et al. Postoperative antibiotic therapy for children with perforated appendicitis: long course of intravenous antibiotics versus early conversion to an oral regimen. Am J Surg. 2008;195(2):141-143.

16. Peltola H, Unkila-Kallio L, Kallio MJ. Simplified treatment of acute staphylococcal osteomyelitis of childhood. The Finnish Study Group. Pediatrics. 1997:99(6):846-850.

17. Peltola H, Pääkkönen M, Kallio P, Kallio MJ; Osteomyelitis-Septic Arthritis Study Group. Short- versus long-term antimicrobial treatment for acute hematogenous osteomyelitis of childhood: prospective, randomized trial on 131 culture-positive cases. Pediatr Infect Dis J. 2010;29(12):1123-1128.

18. Pääkkönen M, Kallio MJT, Kallio PE, Peltola H. Significance of negative cultures in the treatment of acute hematogenous bone 
and joint infections in children. I Ped Infect Dis. 2013;2(2):119125 .

19. Fink CW, Nelson JD. Septic arthritis and osteomyelitis in children. Clin Rheum Dis. 1986;12:423-435.

20. Crary SE, Buchanan GR, Drake CE, Journeycake JM. Venous thrombosis and thromboembolism in children with osteomyelitis. J Pediatr. 2006;149(4):537-541.
21. Gonzalez BE, Teruya J, Mahoney DH Jr, et al. Venous thrombosis associated with staphylococcal osteomyelitis in children. Pediatrics. 2006;117(5):1673-1679.

22. Pickering LK, Baker CJ, Kimberlin DW, Long SS. Red Book: 2009 Report of the Committee on Infectious Diseases. 28th ed. Elk Grove Village, IL: American Academy of Pediatrics; 2012. 